\title{
A Comparative Retrospective Analysis of Mortality, Renal Dysfunction, and Incidence of Bleeding in Patients Receiving Hydroxyethyl Starch 130/0.4 (HES 130/0.4) or Albumin
}

\author{
Matthew Blackburn, Patrick Ratliff, Marintha Short \\ Department of Pharmacy Services, Saint Joseph Hospital, Lexington, USA \\ Email: matt.blackburn3@gmail.com
}

Received 12 June 2014; revised 12 July 2014; accepted 12 August 2014

Copyright (C) 2014 by authors and Scientific Research Publishing Inc.

This work is licensed under the Creative Commons Attribution International License (CC BY).

http://creativecommons.org/licenses/by/4.0/

C. (†) Open Access

\section{Abstract}

Background: In June 2013, the FDA issued a Black Box Warning on synthetic colloids resulting from data indicating an increased risk of mortality, renal injury, and excess bleeding incidence in septic patients admitted to the Intensive Care Unit (ICU), and patients undergoing coronary artery bypass graft (CABG) surgery. The purpose of this study was to compare the incidence of mortality, incidence/severity of renal injury and bleeding in septic ICU patients, and patients undergoing CABG surgery who have received hydroxyethyl starch 130/0.4 or albumin. Methods: This was a retrospective, single center, cohort study conducted at a 433 bed tertiary care hospital in Lexington, Kentucky. Computer generated lists provided patients who received hydroxyethyl starch $130 / 0.4$ or albumin during inpatient stay as either a sepsis patient in the ICU or post coronary artery bypass graft patient. All cause mortality was the primary endpoint and secondary endpoints included overall renal dysfunction, incidence of bleeding, and overall length of stay. Results: A total of 89 patients were evaluated that received albumin or hydroxyethyl starch 130/0.4 alone with either septic ICU or CABG diagnosis codes. There was no significant difference in all cause mortality $(p=0.64)$. Overall renal dysfunction showed no statistically significance between the two groups $(p=0.66)$. There was no statistically significant difference between the albumin or HES 130/0.4 arms with respect to bleeding (TIMI Minimal $p=0.57$, TIMI Minor $p=0.69$, TIMI Major $p=$ 0.35). Patient who received albumin had a statistically significant longer hospital overall length of stay $(p=0.003)$. Conclusion: There was no difference between HES $130 / 0.4$ and albumin in mortality, renal dysfunction, and bleeding in septic ICU and CABG patients. Patients receiving albumin had an increased overall length of stay. These findings suggest that hydroxyethyl starch 130/0.4 may be safe in the studied patient populations; however, further investigation into specific HES 


\title{
agents should be conducted.
}

\section{Keywords}

\author{
Hydroxyethyl Starch, HES, Albumin, Renal Dysfunction, RIFLE
}

\section{Background}

Hypovolemia is a state of decreased blood volume, specifically plasma. Of individuals admitted to the hospital to an inpatient status, septic intensive care unit (ICU) and coronary artery bypass graft (CABG) patients may be commonly affiliated with a state of hypovolemia. In order to achieve a euvolemic state, crystalloid or colloid therapies can be utilized. Common crystalloid therapies administered in this setting are $0.9 \%$ Sodium Chloride ( $0.9 \%$ NS) and Lactated Ringers (LR). Should crystalloid therapy not be desired, or is ineffective, hydroxyethyl starch 130/0.4 (HES 130/0.4), hetastarch, and albumin are colloids that may be used in hypovolemic patients to restore intravascular volume [1] [2].

Hetastarch was initially used as an alternative to human albumin (HA) in a state of hypovolemia. Hetastarch has a large molecular weight, on average 670,000 daltons, as well as a molar substitution of 0.75 which has proven to significantly contribute to the adverse effect profile of renal impairment and coagulopathy [1]. This high molecular weight decreases the ability of the endogenous $\alpha$-amylase enzyme to metabolize hetastarch into a lower molecular weight that the kidney can more effectively and efficiently eliminate [2]. A large molar substitution is a key component to the adverse effect profile as this correlates with the water solubility of the molecule. The larger the molar substitution the less water soluble resulting in decreased renal elimination [3]. Upon initial FDA approval in 2007, HES 130/0.4 provided physicians with an alternative to hetastarch for volume expansion providing a smaller molecular weight of 130,000 daltons and a lower molar substitution of 0.4 . To gain FDA approval, the manufacturer presented data from two separate clinical trials. The first trial compared HES 130/0.4 to hetastarch in orthopedic surgery patients, and the second trial compared HES 130/0.4 to NS in severe sepsis patients. In the trial observing orthopedic patients, HES 130/0.4 demonstrated equivalent efficacy for intraoperative volume expansion when compared to hetastarch, and had a significantly lower effect on coagulation factors (von Willebrand factor, Factor VIII, and Ristocetin cofactor). In the analysis of severe sepsis patients, where HES 130/0.4 was compared to normal saline, patients receiving HES 130/0.4 received less overall volume to correct hypovolemia. As a result of these two trials, hydroxyethyl starch 130/0.4 was marketed to have decreased adverse effect profile when compared to hetastarch. Despite the evidence presented to the FDA upon approval, major safety concerns of all hydroxyethyl starch products continue including coagulation abnormalities, acute kidney injury/renal failure, and refractory pruritus which are generally in the post operative setting [2] [3].

Albumin is a natural colloid that can also be used in the setting of hypovolemia. Albumin has a molecular weight of 66,500 daltons. In the Saline verses Albumin Fluid Evaluation (SAFE) study, HA was found to be equally as effective as normal saline for volume expansion. However, this study also found that human albumin had an increased mortality rate in patients in the ICU setting with severe sepsis. It was also noted that the use of HA was associated with an increased incidence of renal dysfunction (increasing serum creatinine levels and need for dialysis up to two fold) [4].

In June of 2013, the FDA has issued a Black Box Warnings regarding hydroxyethyl starch products because of data indicating an increased risk of mortality and renal injury; as well as, excess bleeding incidence in patients receiving these products. The data indicating increased risk of mortality and renal injury were observed in patients admitted to the ICU, particularly ICU patients with sepsis. Excess bleeding incidence was discovered in patients undergoing open heart surgery in association with cardiopulmonary bypass due to the negative effects HES has shown on coagulation factors. The FDA indicated that these effects could be seen up to 90 days after administration of these products [5].

The FDA made these recommendations for use of HES solutions based upon three distinct trials that compared HES to crystalloid therapy in patients with severe sepsis and septic shock: the CRYSTMAS trial, the 6S trial, and the CHEST trial [6]-[8]. In addition to the FDA recommendations, there have been numerous pub- 
lished articles that debate if colloids are in fact superior to crystalloids for volume expansion. Multiple metaanalyses were conducted that support the FDA findings concluding that synthetic colloids, specifically HES, are associated with increased risk of renal failure when given to critically ill patients with sepsis [9] [10].

In light of these warnings, as well as numerous literature sources recommending that HES 130/0.4 no longer be used as a volume expansion agent in specific patient populations, this study was designed to compare the safety profile of HES 130/0.4 to albumin by analysis of inpatient mortality, kidney injury according to Risk, Injury, Failure, Loss, End Stage Disease (RIFLE) criteria, incidence of hemorrhage after HES 130/0.4 administration, and severity of hemorrhage.

\section{Methods}

This is a retrospective, single center cohort study of matched patients who had an ICD-9 code of post CABG or an ICD-9 of sepsis in the ICU setting of a 433 bed tertiary care center that completes approximately 1000 CABG procedures annually. This study was conducted observing patients with these two distinct ICD-9 codes from June 2012 to June 2013, and was granted exempt status by the Western Institutional Review Board prior to data collection. An electronic database was surveyed for patients with the solitary ICD-9 diagnosis code for sepsis or CABG separately, who received HES 130/0.4 or albumin during their inpatient stay. Patient charts and electronic medical records were surveyed to evaluate patient specific parameters to determine mortality, RIFLE criteria, need for renal replacement therapy, and major or minor bleeding according to the modified Thrombolysis In Myocardial Infarction (TIMI) Study Group bleed criteria.

The primary endpoint of this study was defined as a composite of all-cause mortality. Secondary endpoints included the incidence and degree of acute kidney injury in accordance to RIFLE criteria, the incidence and degree of hemorrhage, and overall length of stay. Baseline lab values were established using the first recorded labs collected for the patient upon admission. Degree of kidney injury according to RIFLE criteria was determined by calculation of change in serum creatinine from baseline or decrease in urine output from baseline. The degree of hemorrhage in accordance to TIMI bleeding criteria was determined by calculating decreases in hemoglobin and/or hematocrit levels in association with any overt sign of hemorrhage or intracranial hemorrhage.

Patients who were considered for inclusion for this study were 18 years of age or older, had a separate inpatient diagnosis of sepsis while in the ICU or CABG identified by International Classification of Diseases, 9th Edition, Clinical Modification (ICD-9) Codes, and received either HES or albumin. Patients were excluded if complications developed greater than 90 days after administration of one of the suspected agents, non-septic ICU or non-CABG patients, received HES and albumin, or if they had pre-existing renal dysfunction (as defined by a calculated creatinine clearance of less than $15 \mathrm{~mL} / \mathrm{min}$ as calculated by Cockroft-Gault or requiring renal replacement therapy) upon admission. Patients were excluded if they received both HES 130/0.4 and albumin due to the fact that the offending agent could not be determined in this circumstance. Prison inmates were also excluded for this study.

\section{Statistical Analysis}

Descriptive statistics for all demographic data was analyzed using electronic statistical software within Microsoft Excel. Continuous variables were analyzed utilizing the student's t-test. Categorical data was examined using the Fisher's exact and Chi-square tests. A p-value $<0.05$ was considered statistically significant for all tests.

\section{Results}

An electronic survey revealed 2330 patients from June 2012-June 2013 who had the distinct ICD-9 diagnosis code for sepsis or the sole ICD-9 diagnosis code for CABG. From these 2330 patients who had an ICD-9 code of sepsis or a separate ICD-9 code of CABG, 583 patients composed the study cohort meeting inclusion criteria as being greater than 18 years of age, if septic then within the ICU setting, post-CABG, and received either albumin or HES 130/0.4. From the 583 patients making up the cohort, patients who received HES 130/0.4 $(n=36)$ or albumin $(n=53)$ alone were included in the study. Patients who received HES 130/0.4 and albumin in combination during admission were excluded from the cohort at this time. Due to time constraints, of the 583 patient meeting inclusion criteria data was collected and analyzed on 89 patients.

When observing baseline characteristics (Table 1) there was no statistically significant variance between the 
Table 1. Baseline characteristics.

\begin{tabular}{lccc}
\hline & Albumin $(\mathrm{n}=53)$ & HES $(\mathrm{n}=36)$ & p-Value \\
\hline Age (years) & $65.46(11.94)$ & $61.22(9.86)$ & 0.08 \\
Sex & & & 0.83 \\
Male (\%) & $35(66)$ & $23(63.9)$ & 0.69 \\
Female (\%) & $18(34)$ & $13(36.1)$ & 0.72 \\
Height (cm) & $172.36(10.32)$ & $171.59(9.39)$ & 0.25 \\
Weight (kg) & $84.61(19.21)$ & $89.72(21.73)$ & 0.11 \\
Body Mass Index & $28.31(5.30)$ & $30.29787(6.08)$ & 0.38 \\
Body Surface Area (m²) & $2.00(0.27)$ & $2.05(0.28)$ & 0.45 \\
Serum Creatinine (mg/dL) & $0.97(0.39)$ & $0.90(0.40)$ & 0.06 \\
Creatinine Clearance: Cockroft-Gault (mL/min) & $83.27(34.48)$ & $97.45(35.78)$ & 0.12 \\
Hemoglobin (g/dL) & $11.73(2.42)$ & $12.5(1.97)$ & 0.27 \\
Hematocrit (\%) & $35.99(6.75)$ & $37.5(5.3)$ & \\
\hline
\end{tabular}

Data is in mean (+/-SD) or N(\%).

HES 130/0.4 ( $n=36)$ and albumin $(n=53)$ arms after analysis with a student's t-test. Patients observed were on average in their early 60's in terms of age. Greater than $50 \%$ of patients in both arms were male with a baseline serum creatinine $(\mathrm{Scr})$ of $<1 \mathrm{mg} / \mathrm{dL}$ and creatinine clearance $(\mathrm{CrCl})$ of $>80 \mathrm{~mL} / \mathrm{min}$. For the primary outcome of all cause mortality, two patients in the albumin arm (3.8\%) and one in the HES arm (2.8) passed away (p = 0.64) (Table 2).

In analysis of renal dysfunction, both deviation from baseline serum creatinine and urine output (UOP) levels were calculated (meaning any decrease in Scr or UOP from baseline). Of those with deviation, 41 albumin (77.4\%) patients and 31 HES 130/0.4 (86.1\%) had alterations in serum creatinine less than five days after administration ( $\mathrm{p}=0.30)$. This observation was also seen for deviation from baseline UOP, as 46 albumin (86.8\%) and 35 HES 130/0.4 (97.2\%) patients saw changes in urine output after administration ( $=0.09)$. Complete deviation from baseline serum creatinine and urine output by time frame is outlined in Table 3 and Table 4 . When stratifying patients to determine if RIFLE criteria were met from the extent of deviation, 26 albumin (49.1\%) and 16 HES 130/0.4 (44.4\%) patients met qualification for renal dysfunction (RIFLE criteria is outlined in definitions). These numbers indicate that there was no statistically significant difference between albumin and HES with respect to renal dysfunction $(\mathrm{p}=0.66)$ (Table 5).

When observing the incidence and severity of bleeding by TIMI criteria, 34 albumin (64.2) and 21 HES 130/0.4 (58.3) patients met criteria for minimal bleeds ( $p=0.58)$. For minor bleeds 17 albumin (32.1) and 13 HES 130/0.4 (36.1) met criteria ( $p=0.69)$. Only 2 albumin (3.8) and 2 HES 130/0.4 (5.6) patients met criteria to be categorized as major bleeds $(\mathrm{p}=0.36)$. Statistics for TIMI criteria bleeds can be seen in Table 6.

In terms of overall hospital length of stay (LOS), patients who received albumin had an average length of stay of 11.46 days, compared to those receiving HES 130/0.4 which had an average LOS of 6.08 days $(p=0.003)$ (Table 7).

\section{Discussion}

The FDA developed their recommendations for use of HES 130/0.4 solutions based upon three distinct trials that compared HES 130/0.4 to crystalloid therapy in patients with severe sepsis and septic shock. The CRYSTMAS trial compared hydroxyethyl starch solution to 0.9\% Sodium Chloride for fluid resuscitation. The CRYSTMAS trial produced results that showed no difference in mortality between the two agents [6]. However, this study was not adequately powered for the results presented. The 6S trial observed severe sepsis patients who received hydroxyethyl starch solution or Lactated Ringer. The 6S trial showed that patients receiving hydroxyethyl starch 
Table 2. Mortality.

\begin{tabular}{cccc} 
& Albumin $(\mathrm{n}=53)$ & HES $(\mathrm{n}=36)$ & $\mathrm{p}-\mathrm{Value}$ \\
\hline All Cause Mortality & $2(3.8)$ & $1(2.8)$ & 0.64 \\
\hline
\end{tabular}

Data is in mean $(+/-\mathrm{SD})$ or $\mathrm{N}(\%)$.

Table 3. Renal dysfunction (Scr).

\begin{tabular}{cccc}
\hline Time Frame (Days) & Albumin $(\mathrm{n}=53)$ & HES $(\mathrm{n}=36)$ & p-Value \\
\hline$<5$ Days & $41(77.4)$ & $31(86.1)$ & 0.30 \\
5 - 9 Days & $3(5.7)$ & $1(2.8)$ & 0.46 \\
$10-19$ Days & $3(5.7)$ & $2(5.6)$ & 0.68 \\
$20-29$ Days & $2(3.8)$ & $1(2.8)$ & 0.64 \\
$\geq 30$ Days & $1(1.9)$ & $0(0)$ & 0.59 \\
\hline
\end{tabular}

Data is in mean (+/- SD) or N(\%); Renal Dysfunction Classified by RIFLE Criteria; \# of days determined from the date of last administered dose of offending agent.

Table 4. Renal dysfunction (UOP).

\begin{tabular}{cccc}
\hline Time Frame (Days) & Albumin $(\mathrm{n}=53)$ & HES $(\mathrm{n}=36)$ & $\mathrm{p}$-Value \\
\hline$<5$ Days & $46(86.8)$ & $35(97.2)$ & 0.09 \\
5 - 9 Days & $2(3.8)$ & $0(0)$ & 0.35 \\
10 - 19 Days & $4(7.5)$ & $1(2.8)$ & 0.32 \\
20 - 29 Days & $0(0)$ & $0(0)$ & 1.00 \\
$\geq 30$ Days & $1(1.9)$ & $0(0)$ & 0.59 \\
\hline
\end{tabular}

Data is in mean (+/- SD) or N(\%); Renal Dysfunction Classified by RIFLE Criteria; \# of days determined from the date of last administered dose of offending agent.

Table 5. Overall renal dysfunction.

\begin{tabular}{cccc} 
& Albumin $(\mathrm{n}=53)$ & HES (n=36) & p-Value \\
\hline Renal Dysfunction (Any) & $26(49.1)$ & $16(44.4)$ & 0.67 \\
\hline
\end{tabular}

Data is in mean (+/- SD) or N(\%); Renal Dysfunction Classified by RIFLE Criteria.

Table 6. Incidence of bleed.

\begin{tabular}{cccc}
\hline & Albumin $(\mathrm{n}=53)$ & HES $(\mathrm{n}=36)$ & p-Value \\
\hline TIMI Minimal & $34(64.2)$ & $21(58.3)$ & 0.58 \\
TIMI Minor & $17(32.1)$ & $13(36.1)$ & 0.69 \\
TIMI Major & $2(3.8)$ & $2(5.6)$ & 0.36 \\
\hline
\end{tabular}

Data is in mean (+/-SD) or N(\%); Renal Dysfunction Classified by TIMI Criteria.

Table 7. Length of stay.

\begin{tabular}{lccc} 
& Albumin $(\mathrm{n}=53)$ & HES $(\mathrm{n}=36)$ & $\mathrm{p}-\mathrm{Value}$ \\
\hline Average Overall LOS (Days) & $11.46(10.19)$ & $6.08(2.91)$ & 0.003 \\
\hline
\end{tabular}

Data is in mean (+/-SD) or N(\%). 
solution had an increased mortality of statistical significance [7]. The CHEST trial compared hydroxyethyl starch solution to $0.9 \%$ Sodium Chloride in ICU patients. The CHEST trial reviled no significant difference in 90-day mortality between patients receiving the agents [8]. In addition to the FDA recommendations, there have been numerous published articles that debate if colloids are in fact superior to crystalloids for volume expansion. Multiple meta-analyses were conducted that support the FDA findings concluding that synthetic colloids, specifically hydroxyethyl starch solutions, are associated with increased risk of renal failure when given to critically ill patients with sepsis [9] [10].

This study challenges the FDA recommendations developed from the CRYSTMAS, CHEST and 6S trials. The hypothesis surrounding this study stated that synthetic colloids other than HES 130/0.4 were the offending agents. This hypothesis was derived due to the fact that many practioners using HES 130/0.4 expressed that they were not observing the same Black Box warnings the FDA published. After data analysis the hypothesis tested was accurate. When looking at all-cause mortality, there was no difference in patients receiving HES 130/0.4 and those who received albumin. This finding refutes the FDA statement that there is increased mortality in patients receiving HES 130/0.4. The results for incidence of renal dysfunction as stratified by RIFLE criteria were of no statistical significance. Within this stratification for renal dysfunction the time frame which a renal injury took place was also recorded. Of interest is that the majority of patients in both observed groups had deviation in Scr and UOP less than five days after receiving a dose of the agent. In both Scr and UOP observations a smaller percentage of patients in the albumin arm had a deviation. While this is not statistically significant it may have a clinical impact. Bleeding, as determined by TIMI criteria, also showed no statistically significant difference in patients receiving HES 130/0.4 versus albumin. These findings contradict the FDA statement that HES 130/0.4 solutions increase the patients' risk of renal dysfunction and bleeding. With respect to the FDA statement regarding observation of the adverse effects of HES 130/0.4 being seen up to 90 days post administration, this study indicated that most patients had an incidence of bleed or alternation in renal function less than five days after administration of the agent. Only one patient showed indication of renal injury $>30$ days after administration with respect to both change in Scr and UOP, and only one patient displayed bleeding $>30$ days post administration (Table 8). Both observations of effects $>30$ days after administration were seen in the albumin arm of the study indicating that only a small percentage if any effects of HES are seen $>30$ days post administration. While this was not of clinical significance between the HES and albumin arms it is noteworthy that this study rarely displayed adverse effects $>30$ days after administration.

This study did identify that patients who received albumin had a significantly longer length of stay than those receiving HES 130/0.4. There were no observed trends or patient specific parameters which would indicate a reason for this outcome.

Our study does have limitations. First, our study was not powered and looked at a small patient population. Second, some patients may have been lost to discharge. Individuals who may have developed complications after discharge yet were still within the 90 day window would not have been detected unless the patient was readmitted to our facility. In addition, patients who received hydroxyethyl starch products other than HES 130/0.4 were excluded. These patients were not included into the study due to only seven patients in the selected date range receiving an agent other than HES 130/0.4. It should be noted that HES 130/0.4 was used significantly more than other HES formulations during this time frame due to formulary adherence by prescribing physicians. Also, patients received either HES 130/0.4 or albumin for their hypovolemic state by physician preference.

Table 8. Decrease in Hg or Hct.

\begin{tabular}{cccc}
\hline Time Frame (Days) & Albumin $(\mathrm{n}=53)$ & HES $(\mathrm{n}=36)$ & $\mathrm{p}$-Value \\
\hline$<5$ Days & $47(88.7)$ & $34(94.4)$ & 0.46 \\
5 - 9 Days & $3(5.7)$ & $0(0)$ & 0.28 \\
10 - 19 Days & $2(3.8)$ & $2(2.8)$ & 0.64 \\
20 - 29 Days & $0(0)$ & $0(0)$ & 1.00 \\
$\geq 30$ Days & $1(1.9)$ & $0(0)$ & 0.99 \\
\hline
\end{tabular}

Data is in mean (+/- SD) or N(\%); Renal Dysfunction Classified by TIMI Criteria; \# of days determined from the date of last administered dose of offending agent. 
There was no observed trend in those with a more severe illness receiving one agent over another.

\section{Conclusion}

With respect to all cause mortality, renal dysfunction, and bleeding, there was no significant difference in patients with the ICD-9 diagnosis code of sepsis in the ICU and patients with the ICD-9 diagnosis code of CABG who received either HES 130/0.4 or albumin. Patients with these specific and unique diagnosis codes, who received albumin, had a statistically significant longer length of stay compared to those administered HES 130/0.4. These findings may warrant further investigation with a larger sample of both ICD-9 groups of septic ICU patients and CABG patients.

\section{References}

[1] Hospira (2008) Hextend (Package Insert).

[2] Hospira (2012) Voluven (6\% Hydroxyethyl Starch 130/0.4 in 0.9\% Sodium Chloride Injection) (Package Insert).

[3] Jungheinrich, C. and Neff, T.A. (2005) Pharmacokinetics of Hydroxyethyl Starch. Clinical Pharmacokinetics, 7, 681699. http://dx.doi.org/10.2165/00003088-200544070-00002

[4] Boldt, J. (2010) Use of Albumin: An Update. British Journal of Anaesthesia, 104, 276-284. http://dx.doi.org/10.1093/bja/aep393

[5] FDA (2013) Hydroxyethyl Starch Solutions: FDA Safety Communication-Boxed Warning on Increased Mortality and Severe Renal Injury and Risk of Bleeding. FDA MedWatch.

[6] Guidet, B., Martinet, O., Boulain, T., Philippart, F., Poussel, J.F., Maizel, J., Forceville, X., Feissel, M., Hasselmann, M., Heininger, A. and Van Aken, H. (2012) Assessment of Hemodynamic Efficacy and Safety of 6\% Hydroxyethylstarch $130 / 0.4$ vs. $0.9 \% \mathrm{NaCl}$ Fluid Replacement in Patients with Severe Sepsis: The CRYSTMAS Study. Critical Care, 16, R94. http://dx.doi.org/10.1186/cc11358

[7] Perner, A., Haase, N., Guttormsen, A.B., Tenhunen, J., Klemenzson, G., Åneman, A., Madsen, K.R., Møller, M.H., Elkjær, J.M., Poulsen, L.M., Bendtsen, A., Winding, R., Steensen, M., Berezowicz, P., et al. (2012) Scandinavian Critical Care Trials Group. Hydroxyethyl Starch 130/0.42 versus Ringer's Acetate in Severe Sepsis. New England Journal of Medicine, 367, 124-134. http://dx.doi.org/10.1056/NEJMoa1204242

[8] Myburgh, J.A., Finfer, S., Bellomo, R., Billot, L., Cass, A., Gattas, D., Glass, P., Lipman, J., Liu, B., McArthur, C., McGuinness, S., Rajbhandari, D., Taylor, C.B. and Webb, S.A. (2012) Australian and New Zealand Intensive Care Society Clinical Trials Group. Hydroxyethyl Starch or Saline for Fluid Resuscitation in Intensive Care. New England Journal of Medicine, 367, 1901-1911. http://dx.doi.org/10.1056/NEJMoa1209759

[9] Hartog, C.S., Bauer, M. and Reinhart, K. (2011) The Efficacy and Safety of Colloid Resuscitation in the Critically Ill. Anesthesia \& Analgesia, 112, 156-164. http://dx.doi.org/10.1213/ANE.0b013e3181eaff91

[10] Zarychanski, R., TA, Fergusson, D.A., Cook, D.J., Hebert, P., Bagshaw, S.M., Monsour, D. and McIntyre, L.A. (2009) Renal Outcomes and Mortality Following Hydroxyethyl Starch Resuscitation of Critically Ill Patients: Systematic Review and Meta-Analysis of Randomized Trials. Open Medicine, 3, E196-E209.

[11] TIMI.org. TIMI Definitions for Commonly Used Terms in Clinical Trials.

[12] Hoste, E.A.J., Clermont, G., Kersten, A., et al. (2006) RIFLE Criteria for Acute Kidney Injury Are Associated with Hospital Mortality in Critically Ill Patients: A Cohort Analysis. Critical Care, 10, R73.

http://dx.doi.org/10.1186/cc4915 


\section{Abbreviations}

ICU-Intensive Care Unit

CABG-Coronary Artery Bypass Graft

HES 130/0.4-Hydroxyethyl Starch 130/0.4

TIMI-Thrombolysis in Myocardial Infarction

RIFLE-Risk, Injury, Failure, Loss, End-Stage Renal Dysfunction

FDA-Food and Drug Administration

ICD 9-International Classification of Diseases, 9th Edition, Clinical Modification (ICD-9-CM) Codes

Scr-Serum Creatinine

CrCl-Creatinine Clearance

UOP_Urine Output

LOS-Length of Stay

\section{Definitions}

- $\quad$ TIMI criteria [11]

○ Major

$\square \quad$ Intracranial hemorrhage or a $>5 \mathrm{~g} / \mathrm{dL}$ decrease in hemoglobin concentration or a $>15 \%$ absolute decrease in hematocrit

$\square$ If CABG related: fatal bleeding or perioperative intracranial bleeding or transfusion of $\geq 5$ units of whole blood or PRBCs within 48 hour period or chest tube output $>2$ L within a 24 hour period.

○ Minor

$\square$ Any overt sign of hemorrhage associated with a fall in hemoglobin 3 to $\leq 5 \mathrm{~g} / \mathrm{dL}$ or when hemoglobin is not available a $9 \%$ to $\leq 15 \%$ decrease in hematocrit.

- Minimal

$\square$ Any overt sign of hemorrhage associated with a fall in hemoglobin $<3 \mathrm{~g} / \mathrm{dL}$ or a fall of hematocrit $<$ $9 \%$ when hemoglobin is not available

- RIFLE criteria [12]

○ Risk

$\square$ Scr increase $1.5 x$ baseline or decrease in urine output of $<0.5 \mathrm{~mL} / \mathrm{kg} / \mathrm{hr}$ for 6 hours

○ Injury

$\square$ Scr $2 x$ baseline or decrease in urine output of $<0.5 \mathrm{ml} / \mathrm{kg} /$ hour $\times 12$ hours

- Failure

$\square$ Scr $3 x$ baseline, or Scr $\geq 4 \mathrm{mg} / \mathrm{dl}$ with an acute rise $>0.5 \mathrm{mg} / \mathrm{dl}$ or a decrease in urine output $<0.3$ $\mathrm{ml} / \mathrm{kg}$ /hour $\times 24$ hours, or anuria $\times 12$ hours

○ Loss

$\square$ Persistent acute renal failure $=$ complete loss of kidney function $>4$ weeks

- End-stage kidney disease

$\square \quad$ End-stage kidney disease $>3$ months 
Scientific Research Publishing (SCIRP) is one of the largest Open Access journal publishers. It is currently publishing more than 200 open access, online, peer-reviewed journals covering a wide range of academic disciplines. SCIRP serves the worldwide academic communities and contributes to the progress and application of science with its publication.

Other selected journals from SCIRP are listed as below. Submit your manuscript to us via either submit@scirp.org or Online Submission Portal.
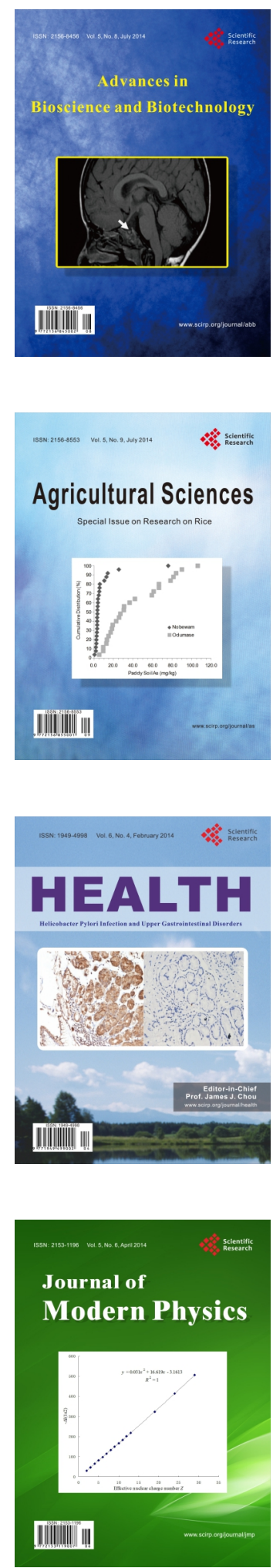
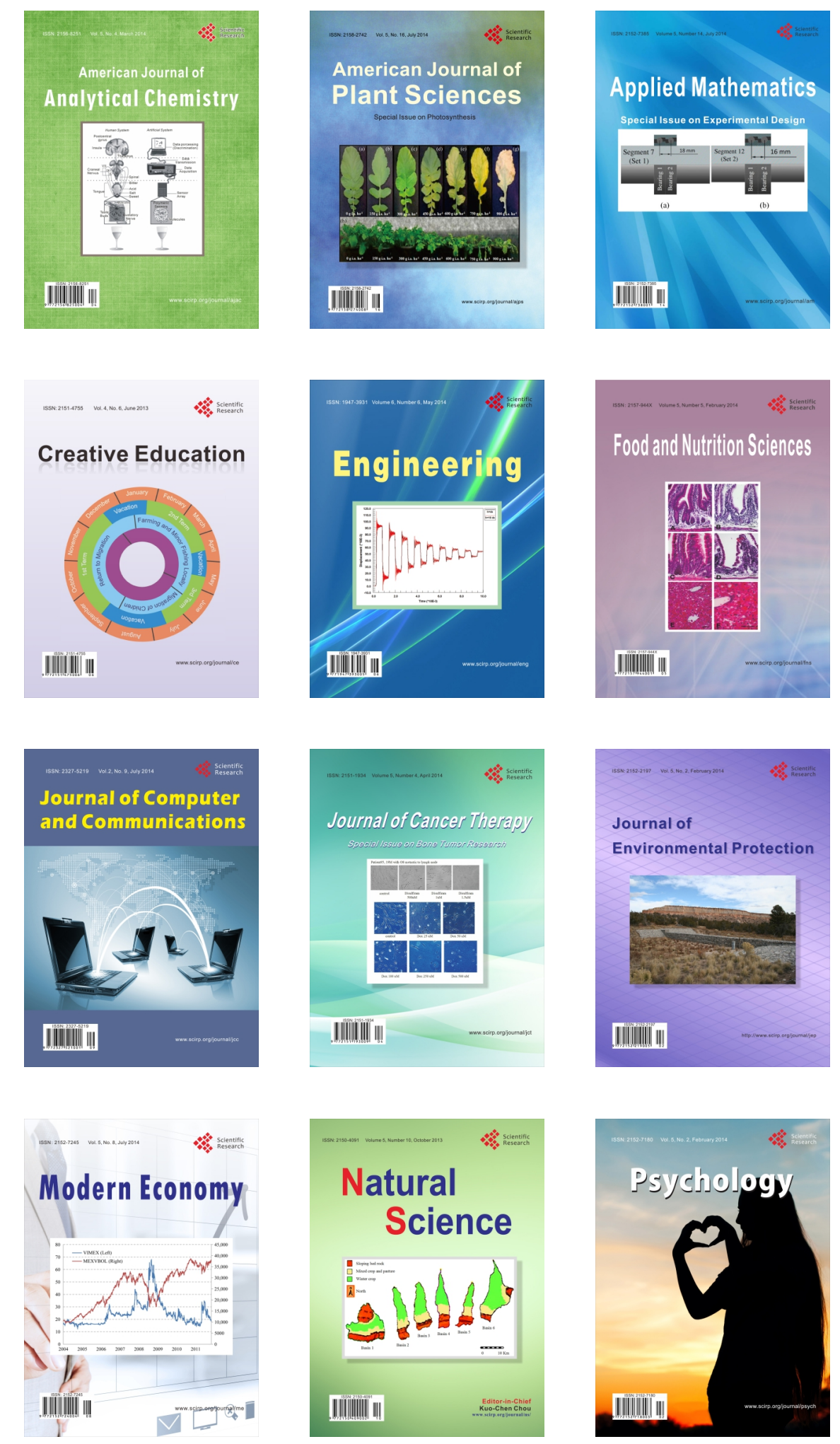